Published in final edited form as:

Science. 2007 August 10; 317(5839): 757-758.

\title{
Shining Light on Depression
}

\author{
Thomas R. Insel, M.D.
}

Just as research during the Decade of the Brain (1990-2000) forged the bridge between the mind and the brain, research in the current decade is helping us to understand mental illnesses as brain disorders. As a result, the distinction between disorders of neurology (e.g., Parkinson's and Alzheimer's diseases) and disorders of psychiatry (e.g., schizophrenia and depression) may turn out to be increasingly subtle. That is, the former may result from focal lesions in the brain, whereas the latter arise from abnormal activity in specific brain circuits in the absence of a detectable lesion. As we become more adept at detecting lesions that lead to abnormal function, it is even possible that the distinction between neurological and psychiatric disorders will vanish, leading to a combined discipline of clinical neuroscience (1).

But before we can understand depression as a brain disorder, we need information on the specific neuronal circuits that contribute to the hopeless despair that forms the core of this illness. Neuroimaging studies of people with depression might be helpful for identifying brain regions of interest, but the temporal and spatial resolution of current functional magnetic resonance imaging and positron emission tomography may not capture the real-time dynamics of brain function that are most relevant to mood and cognition. In a new approach, Airan et al. report on page 819 of this issue the use of optical imaging to capture cellular activity at millisecond resolution in brain slices (2). Their study, which uses rodents with some of the behavioral features of depression, does not define the neurobiology of depression in humans, but it demonstrates how optical imaging — in this case, using voltage-sensitive dyes - can identify changes in brain activity, enabling correlations between real-time cellular activity and changing affective state.

The findings of Airan et al. are consistent with other results that implicate the hippocampus in rodent studies of depression. Chronic or intense stressors, such as social defeat, result in behaviors that resemble human depression, and these stressors have been reported to reduce hippocampal neurogenesis (3). They also down-regulate the hippocampal expression of brainderived neurotrophic factor (4), a molecule that promotes neuron survival, proliferation, and differentiation. Clinically effective antidepressants increase hippocampal neurogenesis (5), and blocking neurogenesis during treatment prevents the antidepressant effect in rodents (6).

What about the hippocampus and human depression? Major depressive disorder is associated with cognitive deficits and dysregulation of the hypothalamic-pituitary-adrenal (HPA) axis, part of the neuroendocrine system that controls the stress response. Because the hippocampus is involved in both forming new memories and regulating the HPA axis, one might expect a link between depression and the hippocampus. Indeed, some human neuroimaging studies have reported a subtle reduction in the size of the hippocampus in patients with depression (7), and postmortem studies have reported alterations in hippocampal gene expression (8). But the evidence thus far is unconvincing. Humans with hippocampal lesions have memory deficits but not mood disorders (9). And none of the imaging or postmortem findings have been shown to be specific to the hippocampus or to major depressive disorder. Although the absence of evidence is hardly evidence of absence, most recent clinical studies of the neurobiology of depression have been following a different lead.

Neuroimaging studies of humans with major depressive disorder have largely pointed to prefrontal sites, especially implicating an area in the midline subgenual anterior cingulate cortex, often denoted as area $25(10,11)$. Not only does this region appear abnormal on 
structural and functional scans $(10,11)$, but also it is enriched with the serotonin transporter, a target for many antidepressant drugs. Individuals inheriting a risk allele within the promoter of the serotonin transporter gene have reduced volume of area 25 and reduced functional coupling of this region to the amygdala, a subcortical region implicated in the regulation of emotion (12). An initial study of treatment-resistant depressed patients reports that deep brain stimulation adjacent to area 25 relieves the symptoms of major depressive disorder (13).

How do we resolve the differences between rodent studies that implicate the hippocampus and human studies that implicate the midline prefrontal cortex? Of course, the discrepancies might be attributed to neuroanatomical differences between rodent and human brains. Rodents have at most a primitive subgenual anterior cingulate cortex, whereas this region in the primate brain shows extensive connections with subcortical and cortical targets (14). But other fundamental issues should be kept in mind when jumping from studies of rodent behavior to human psychopathology. Human psychiatric disorders are complicated amalgams of affective, cognitive, and behavioral abnormalities. We might model aspects of one of these dimensions, such as helplessness or memory loss, in rodents; but we are then studying an aspect of the disorder, not the disorder itself.

Major depressive disorder, the result of an unfortunate convergence of genetic and environmental factors, is certainly more than the sum of its observable parts. Identifying brain regions correlated with "the parts" will be an important next step for human imaging studies, but the field will need to avoid high-tech phrenology. Understanding the neurobiology of abnormal mood regulation will not be accomplished through the identification of a focal lesion or a single explanatory hot spot. The task will be to define altered activity within a functional neuronal network that might well include both the ventral hippocampus and midline prefrontal cortex (15). The importance of the new report by Airan et al. is the demonstration of abnormal network dynamics in a defined circuit through the use of a technique with combined spatial and temporal resolution that we have not even begun to consider for human studies. We are not able to apply voltage-sensitive dye imaging to people with major depressive disorder, but studies in model animals that help us to link behavior to real-time circuit information will be the foundation for understanding depression as a brain disorder.

\section{References}

1. Insel TR, Quirion R. J Am Med Assoc 2005;294:2221.

2. AiranRDScience3178192007 [PubMed: 17615305]published online 5 July 2007 (10.11126/science/ 1144400). (See NIMH Science Update --http://www.nimh.nih.gov/press/depressioncrossroads.cfm)

3. Sapolsky RM. Arch Gen Psychiatry 2000;57:925. [PubMed: 11015810]

4. Tsankova N, et al. Nat Neurosci 2006;9:519. [PubMed: 16501568]

5. Duman RS. Biol Psychiatry 2004;56:140. [PubMed: 15271581]

6. Santarelli L, et al. Science 2003;301:805. [PubMed: 12907793]

7. Neumeister A, et al. Biol Psychiatry 2005;57:935. [PubMed: 15820716]

8. Lopez-Figueroa AL, et al. Biol Psychiatry 2004;55:225. [PubMed: 14744462]

9. Squire LR, Stark CEL, Clark RE. Annu Rev Neurosci 2004;27:279. [PubMed: 15217334]

10. Drevets WC, et al. Nature 1997;386:824. [PubMed: 9126739]

11. Mayberg HS, et al. Neuroreport 1997;8:1057. [PubMed: 9141092]

12. Pezawas L, et al. Nat Neurosci 2005;8:828. [PubMed: 15880108]

13. Mayberg HS, et al. Neuron 2005;45:651. [PubMed: 15748841]

14. Ongur D, Price JL. Cereb Cortex 2000;10:206. [PubMed: 10731217]

15. Houenou J, et al. Mol Psychiatry. 200710.1038/sj.mp.4002010 